\title{
Knowledge, Attitude and Awareness of Hand Hygiene Practices amongst Dental Professionals of Central Vidarbha Region of Maharashtra, India
}

Rohit $\mathbf{M}^{*}$ and Alka MD
Department of Oral Pathology\& Microbiology, VSPM's Dental College \& Research Center, India

*Corresponding author: Rohit Moharil, Assistant professor, Department of Oral Pathology\& Microbiology, VSPM's Dental College \& Research Center, India, Tel: 9970171031; Email: drrohitmoharil@gmail.com

\section{Research Article}

Volume 4 Issue 1

Received Date: April 09, 2020

Published Date: June 12, 2020

DOI: $10.23880 /$ phoa-16000154

\section{Abstract}

Aim: To study the knowledge, attitude and awareness about Hand hygiene practices amongst dental professionals of central vidarbha region of Maharashtra India.

Material \& Methods: A questionnaire based survey was carried out on 150 dental professionals in which 20 questions about knowledge, attitude and awareness about Hand hygiene was asked and data was interpreted.

Results: Number of health care professionals included in the study was 150. In the present study the knowledge on hand hygiene was moderate $(41.38 \%)$. It was observed that the average awareness regarding the positive indications of hand hygiene was $49.1 \%$ and only $38.33 \%$ practices the hand hygiene protocol.

Conclusion: Our study highlights the urgent need for introducing measures in order to increase the knowledge, attitudes, practices Teaching Hospital, which may play a very important role in hand hygiene compliance among the staff and reducing cross transmission of infections among patients.

Keywords: Hand Hygiene; Infection

Abbreviations: HH: Hand Hygiene; CDC: Centre for Disease Control; WHO: World Health Organization.

\section{Aim}

To study the knowledge, attitude and awareness about Hand hygiene practices amongst dental professionals.

\section{Introduction}

Dentistry has a responsibility to adhere to scientifically accepted and evidence based principles of infection control. This responsibility is now enforced by an increasing requirement of quality expressed by patients. Hand hygiene $(\mathrm{HH})$ in dental practice is one of the most important parts of the infection control process and is the single most important activity performed to reduce the risk of transmitting microorganisms from provider to patient $[1,2]$. It has been suggested that the optimal duration of hand washing is between 30 seconds and one minute as a minimum and a maximum range respectively. The recommendation on hand hygiene has been updated, and hand washing has been replaced by hand-rub as the standard of care [3]

In the wake of the growing burden of health care associated the infections, increasing severity of illness and complexity of treatment, superimposed by multiple drug resistant pathogenic infections, health care practitioners are reversing back to the basics of infection prevention by simple methods like hand hygiene [4]. Complete guidelines on hand hygiene is given by World Health Organization (WHO) 
and Centre for Disease Control (CDC) [5,6]. To improve the compliance of hand hygiene, in this study the assessment of Knowledge, Attitude and practices of Hand Hygiene in Dental Teaching Institute was done.

\section{Material and Methods}

A questionnaire based survey was carried out on 150 dental professionals in which 20 questions about knowledge, attitude and awareness about Hand hygiene was asked and data was interpreted.

\section{Results}

Number of health care professionals included in the study was 150 . In the present study the knowledge on hand hygiene was moderate (41.38\%). It was observed that the average awareness regarding the positive indications of hand hygiene was $49.1 \%$ and only $38.33 \%$ practices the hand hygiene protocol of the 150, 22 (14.67\%) declared that they had not been received any training regarding $\mathrm{HH}$ (Figures 1-4).
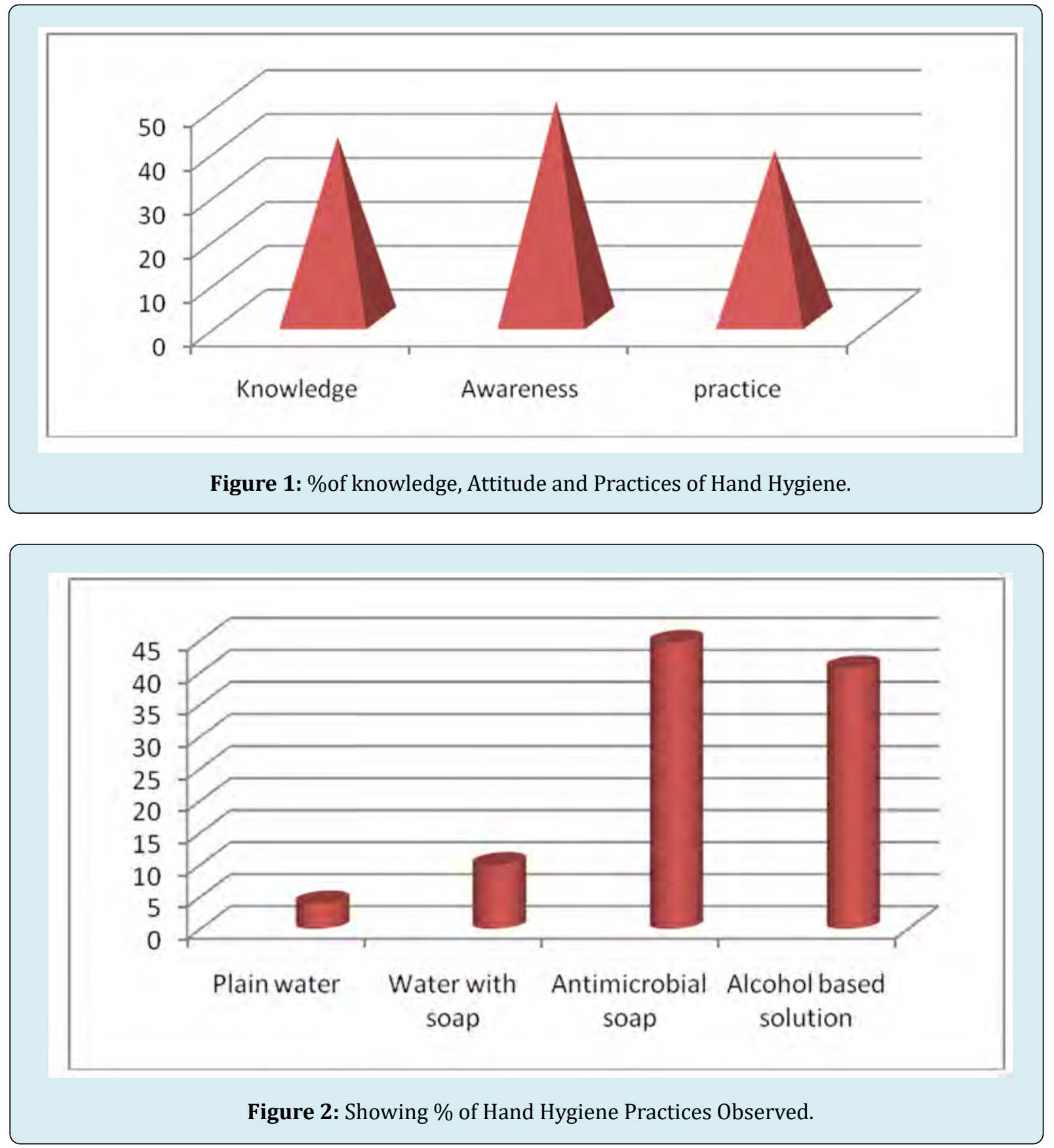


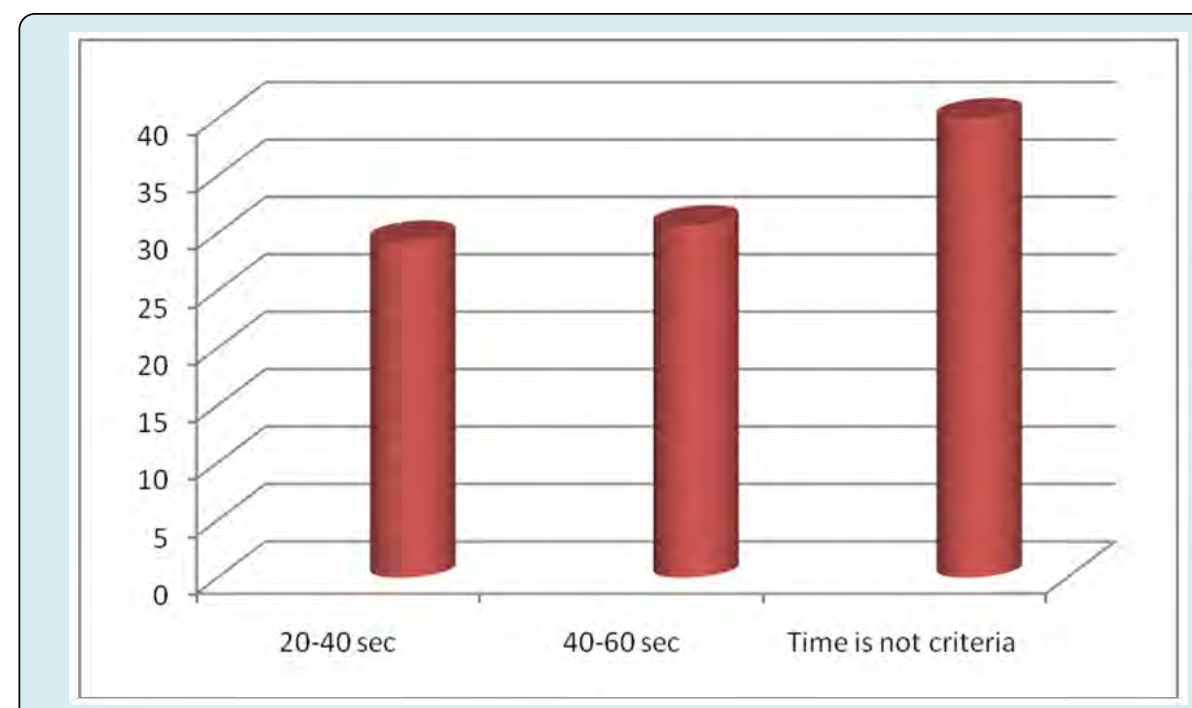

Figure 3: Showing Time Criteria Followed.

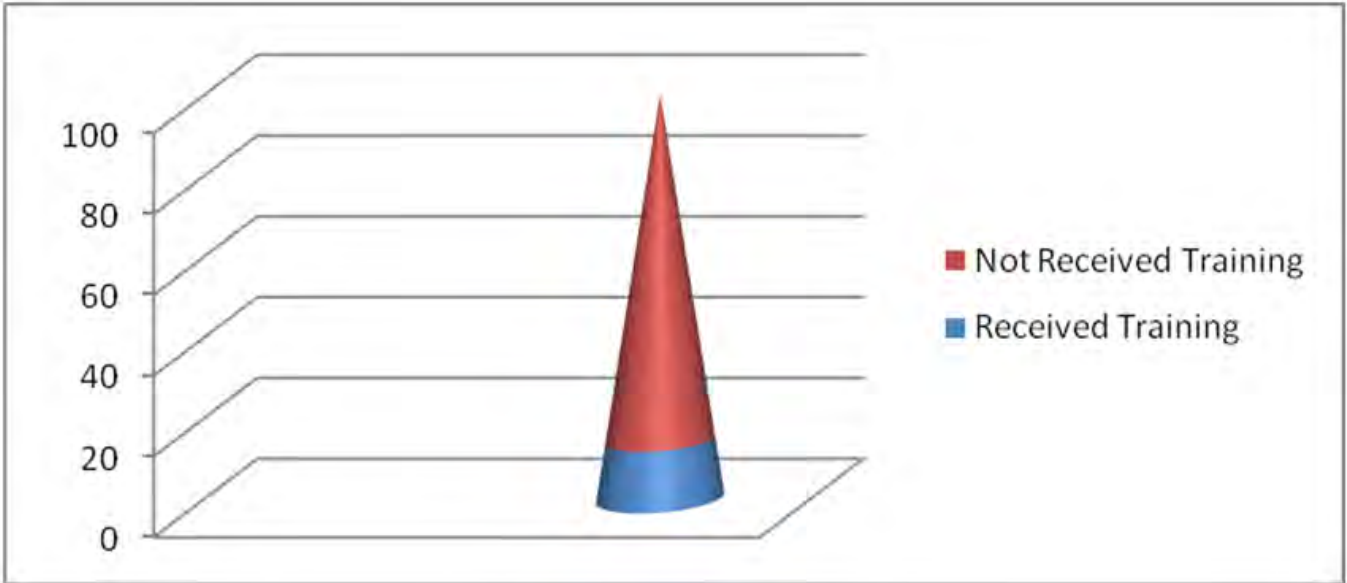

Figure 4: Showing \% of Person Received/Not Received Hand Hygiene Training.

\section{Discussion}

This study was carried out to test the knowledge, attitude and awareness about Hand hygiene practices amongst dental professionals. To author's knowledge, this is the unique study that provides a comprehensive assessment of the $\mathrm{HH}$ practices and beliefs of dental health workers.

In the present study the knowledge on hand hygiene was moderate $(41.38 \%)$. Earlier study from Riyadh, Saudi Arabia by Basurrah M, et al. [3]; among health care workers (HCWs), adherence to hand hygiene was seen among $70 \%$ among medical students, $69.2 \%$ among interns, $18.8 \%$ among nurses, $12.5 \%$ among residents and $9.1 \%$ among consultants.
In our study awareness was $49.1 \%$ but there was lack of proper practices with this knowledge. Kadi, et al. at Saudi Arabia observed that the average awareness regarding the positive indications of hand hygiene was $56 \%$ among medical students [3] indeed; a recent review WHO, et al. [5] reported an average Hand Hygiene compliance of $38.7 \%$ with a baseline ranging from $5 \%$ to $89 \%$. These results are inferior to many studies conducted for dental healthcare workers in countries such as Africa (25\% before glowing and $47 \%$ after), Italy $(55.5 \%)$, Canada (76\%), USA (75\%), and Khartoum state (44\%), United Kingdom (26-63\%). Moreover, it is important to distinguish between studies undertaken in teaching and nonteaching centers, when comparing results.

Different causes of compliance could explain health care professional attitudes toward $\mathrm{HH}$. Interruption in 
care is an important cause of compliance and this frequent situation occurs during treatment sessions. Environmental and personal barriers could explain these iterative breaks. In particular, organization with central dispensing station of all sterile devices enhances the number of persons moving around the clinic and increases the risk of not following the correct $\mathrm{HH}$ protocol [2]. When there was compliance with $\mathrm{HH}$, the results showed a correlation between knowledge note and quality of $\mathrm{HH}$ in our study.

This importance of increasing knowledge about infection control scientific data and about protocols for better compliance was demonstrated by surveys of Cheng, et al. in Taiwan, Cleveland, et al. in US and Hubner, et al. in Germany.

\section{Conclusion}

Our study highlights the urgent need for introducing measures in order to increase the knowledge, attitudes, practices in Teaching institutes \& Hospital, which may play a very important role in hand hygiene compliance among the staff and reducing cross transmission of infections among patients.

\section{References}

1. Ahmed EE (2011) Improving hand hygiene compliance among dental health workers in 3 dental clinics in Khartoum. Thesis Royal College of Surgeons in Ireland, pp: $1-77$.

2. Thivichon PB, Barsotti O, Girard R, Morrier JJ (2014) Hand hygiene practices in a dental teaching center: Measures and improve. Eur J Dent 8(4): 481-486.

3. Mausumi B (2015) Scholars Journal of Applied Medical Sciences 3(6C): 2334-2339.

4. Mohesh G, Dandapani A (2014) Knowledge, attitude and practice of hand hygiene among medical students -A questionnaire based survey. Unique Journal of medical and dental sciences 2(3): 127-131.

5. WHO (2009) WHO guidelines on hand hygiene in health care. World Health Organization, pp: 262.

6. (2020) Hand Hygiene Guidance: Hand Hygiene in Healthcare Settings. Center for disease control and Prevention. 